\section{Prevenção da transmissão vertical do vírus da imunodeficiência humana: análise da adesão às medidas de profilaxia em uma maternidade de referência em Fortaleza, Ceará, Brasil}

\section{Prevention of vertical transmission of HIV: analysis of the adhesion to prophylactic measures in a reference maternity in the city of Fortaleza, State of Ceará, Brasil}

Maria do Socorro Cavalcante 1

Ângela Cristina Bonfim da Silveira 2

Ana Maria de Sousa Ribeiro 3

Alberto Novaes Ramos Júnior 4

1-3 Secretaria de Saúde do Estado do Ceará. Hospital Geral Dr César Cals, Av do Imperador, 545. Fortaleza, CE, Brasil CEP: 60.015-052 .E-mail: socorro.cavalcante@gmail.com 4 Departamento de Saúde Comunitária. Faculdade de Medicina Universidade Federal do Ceará. Fortaleza, CE, Brasil.

\begin{abstract}
Objectives: to analyze the adherence to prophylactic measures implemented in pregnant women for reduction of vertical HIV transmission in this reference maternity in the city of Fortaleza, State of Ceará, Brazil.

Methods: cross sectional study, with descriptive and analytical components. Included were all of the pregnant HIV infected women that attended the maternity during January 2000 to August 2004.

Results: one hundred and ten pregnant HIV infected women were studied and 102 (92.7\%) informed to have frequented prenatal service. Sixty three of them (57.3\%) were informed about their serological status during prenatal, 39 (35.5\%) knew they had Aids before becoming pregnant. It was identified a high percentage of interventions for reduction of $H I V$ vertical transmission, not having statistically difference among women that knew of their serological status the ones that didn't. It is needed to be emphasized that in 86 (78.1\%) cases the adherence to these interventions was considered completed.

Conclusions: the high adherence to conducts for reducing vertical HIV transmission observed in this study reveals the viability of the application of those actions in Fortaleza, nonetheless the specific conditions in this reference hospital doesn't apply to all situations in other services in Fortaleza.
\end{abstract}

Key words Vertical transmission, Human immunodeficiency virus, Maternal and child health

\section{Resumo}

Objetivos: avaliar a adesão às medidas de profilaxia da transmissão vertical do HIV em parturientes infectadas pelo HIV que receberam atendimento em uma maternidade de referência na cidade de Fortaleza, Estado do Ceará, Brasil.

Métodos: estudo transversal, com componentes descritivos e analíticos. Foram incluidas todas as parturientes com infecção pelo HIV admitidas na maternidade no periodo de janeiro de 2000 a agosto de 2004.

Resultados: participaram 110 parturientes infectadas pelo HIV, sendo que 102 (92,7\%) informaram ter freqüentado serviço de pré-natal. Sessenta e três delas $(57,3 \%)$ foram informadas de seu status sorológico no pré-natal, dessas, 39 (35,5\%) sabiam ter Aids antes de engravidar. Identificou-se um elevado percentual de adesão às intervenções para a redução da transmissão vertical do HIV, sem haver diferença estatisticamente significativa entre o grupo de mulheres que conheciam ou não seu status sorológico. Ressalta-se que em 86 $(78,1 \%)$ casos a adesão às intervenções foi considerada completa.

Conclusões: a elevada adesão às condutas para a redução da transmissão vertical do HIV observada nesse estudo revela, por um lado, a viabilidade da aplicação dessas ações em Fortaleza e, por outro, a especificidade das condições de uma maternidade de referência não permitindo inferência sobre a situação global do município.

Palavras-chave Transmissão vertical, Virus da Imunodeficiência Humana, Saúde materno-infantil 


\section{Introdução}

A epidemia da infecção pelo HIV vem evoluindo de forma crescente na população de mulheres em todo o mundo.1,2 No Brasil, dos 362.364 casos de Aids notificados até junho de 2004, 111.314 (30,7\%) ocorreram em mulheres sendo que 92.255 (82,9\%) delas tinham idade entre 20 e 49 anos, faixa etária sabidamente associada com maior capacidade reprodutiva. ${ }^{3}$ Segundo levantamentos realizados em maternidades selecionadas em todo o país, a prevalência estimada da infecção pelo vírus da imunodeficiência humana (HIV) em parturientes, estabelecida por meio de estudos transversais, entre 1997 e 2000, foi de $0,6 \%$ (variando de 0,5 a 1,2\%). ${ }^{1}$ A partir do ano 2000, na cidade de Fortaleza, Ceará, a oferta de testes sorológicos à gestante foi ampliada para unidades de referência em (Doenças Sexualmente Transmissível) e maternidades que dispunham de aconselhamento pré e pós-teste para sorologia antiHIV. Estudos realizados pelo Ministério da Saúde em seis serviços de saúde sentinela encontraram uma prevalência de infecção pelo HIV em parturientes de 0,77\%, em 1997, nível de prevalência mais elevado em relação a um estudo anterior, que mostrou uma prevalência de $0,25 \% .4$

Nesse contexto, a transmissão vertical do HIV tem sido um dos grandes desafios para a saúde pública e representa um dos problemas de maior importância em todo o mundo.1,2 Como conseqüência do crescente número de casos de infecção por HIV entre as mulheres, houve um aumento da transmissão vertical, que no período de 1983 a 1992, era responsável por $64 \%$ dos casos de infecção por HIV em menores de 13 anos, e que aumentou para $83,6 \%$ dos casos, no período de 1993 até junho de 2004.3

$\mathrm{Na}$ ausência de qualquer intervenção, a transmissão vertical do HIV, alcança freqüências de 25,0 a 30,0\%,5-7 dependendo da região estudada. Com a instituição das medidas profiláticas que visam à redução da transmissão vertical é possível alcançar índices tão baixos quanto 1,0 a 2,0\%.2,7 Vários estudos comprovam a eficácia do uso regular de antiretrovirais pela gestante infectada para redução da transmissão vertical, demonstrado pelo protocolo 076 do Aids Clinical Trial Group. ${ }^{8-12}$ Com base nesses estudos, o Ministério da Saúde estabeleceu recomendações e rotinas para unidades de atenção primária e serviços materno-infantis, com ênfase na atenção integral a gestantes e crianças. ${ }^{2,7}$ As orientações desse protocolo para redução da transmissão vertical, publicado desde 1995 e atualizadas em 1997 incluem: oferta universal do teste anti-HIV, juntamente com aconselhamento pré e pós-teste; administração de anti-retrovirais à gestante a partir da $14^{\mathrm{a}}$ semana de gestação e, durante o parto, e ao recém-nascido nas seis primeiras semanas de vida; substituição do aleitamento materno e escolha da via de parto. ${ }^{13-16}$

Em Fortaleza, as recomendações para a redução da transmissão vertical do HIV foram sistematizadas a partir de 2000 e o teste rápido em 2001 em todas as maternidades da rede pública. Em decorrência disso, torna-se de vital importância, avaliar as medidas de prevenção implementadas nas maternidades públicas de Fortaleza que prestam assistência às mulheres infectadas pelo HIV.

Este estudo teve como objetivo analisar a adesão às medidas de prevenção utilizadas em parturientes para a redução da transmissão vertical do HIV em uma maternidade de referência em Fortaleza, Ceará.

\section{Métodos}

O presente estudo foi realizado no Hospital Geral Dr. César Cals que está situado no centro da cidade de Fortaleza, Ceará, Brasil e se constitui em um hospital público, de nível terciário, de referência estadual, com unidade de urgência e emergência em ginecologia e obstetrícia e unidade neonatal. Sua capacidade é de 296 leitos e realiza em média 582 partos por mês. Entre as atividades e projetos desenvolvidos incluem-se: casa da gestante, Projeto Mãe Canguru, Projeto Nascer-Maternidade. É, ainda uma unidade de fácil acesso à população.

$O$ estudo tem delineamento observacional, transversal, com componentes descritivos e analíticos. Foram incluídas no estudo todas as parturientes com infecção pelo HIV admitidas na maternidade, de janeiro de 2000 a agosto de 2004, período que correspondeu ao início das ações de intervenção para a redução da transmissão vertical do HIV no município de Fortaleza, Ceará. Os critérios para inclusão foram: ter resultado reagente pelo teste imunoenzimático (ELISA) e confirmado pela Imunofluorescência Indireta e/ou pelo Western Blot, realizado antes ou durante o pré-natal ou resultado reagente pelo teste rápido (Determine ${ }^{\mathrm{TM}}$ ou Abbott) no momento do parto. Excluíram-se as parturientes admitidas para curetagem ou aborto, por não apresentarem risco de transmissão vertical ao bebê, em um total de cinco no período do estudo, sendo dois abortos e três fetos mortos.

Para esse estudo definiu-se "conhecimento da soropositividade", o momento em que a gestante tomou conhecimento da infecção pelo HIV. Foi classificado em dois grupos: 1) "sabiam ser soroposi- 
tivas antes da gravidez": gestantes que já sabiam ter sorologia positiva para a infecção do HIV ou que já tinham o diagnóstico antes da gravidez; 2) "sabiam ser soropositivas depois da gravidez": gestantes que souberam estar infectadas pelo vírus durante o prénatal ou durante o parto. As informações referentes às parturientes estudadas foram obtidas a partir da revisão dos prontuários médicos utilizando um questionário semi-estruturado. Paralelamente, foram utilizadas outras fontes de informações como a ficha de notificação e investigação epidemiológica da Gestante HIV Positiva e Crianças Expostas do Sistema de Informação de Agravos de Notificação (SINAN) e do Sistema de Informação de Nascidos Vivos (SINASC), para a complementação das informações.

Estabeleceu-se, de forma arbitrária, o valor de $80 \%$ como um bom indicador para avaliar a adesão à realização das medidas profiláticas nas parturientes. Foram considerados neste estudo: a administração de anti-retrovirais (ARV) nos três momentos: durante a gravidez, parto e recém-nascido nas primeiras 24 horas de nascimento, a suspensão do aleitamento materno, bem como, a cesárea eletiva como via de parto. Entendeu-se que, apenas com a adoção dessas cinco medidas profiláticas, já se consegue reduzir a transmissão do vírus. No estudo, não foi levado em consideração o tempo de utilização do ARV durante a gestação devido à falta de informação do início da profilaxia no prontuário e na ficha de investigação; no entanto, foi utilizada apenas a informação da administração do ARV. Considerou-se o termo "adesão completa" quando da realização de todas essas medidas juntas.

Os dados foram analisados utilizando o programa Epi-Info, versão 6.04d, Atlanta, Estados Unidos. Inicialmente foi realizada análise univariada para descrição das características da população do estudo (idade, escolaridade, município de residência, diagnóstico de Aids, realização de pré-natal e registro da utilização de anti-retrovirais pela gestante no pré-natal, parto e recém-nascido). Realizou-se análise multivariada para avaliar os efeitos das variáveis: tipo de parto, profilaxia no pré-natal, profilaxia no parto, profilaxia no recém-nascido, aleitamento, adesão completa entre o grupo de parturientes com "conhecimento da soropositividade" antes ou depois da gravidez. Verificou-se também se escolaridade estava associada à adesão completa das medidas profiláticas. Calculou-se a razão de chances pelo Odds Ratio (OR) sendo o intervalo de confiança de 95\% (IC95\%) e utilizou-se o valor de $\alpha=0,05$ para definir o nível de significância estatística.

O projeto de pesquisa foi submetido e aprovado pelo Comitê de Ética do Hospital Geral Dr. César Cals, onde ocorreu o estudo e faz parte de uma linha de pesquisa do grupo. A confidencialidade, a privacidade e o sigilo das informações das pacientes foram resguardas pelos pesquisadores em todas as etapas do estudo.

\section{Resultados}

Foram incluídas neste estudo 110 parturientes que preencheram os critérios de inclusão, e cinco foram excluídas. A idade das parturientes estudadas variou entre 15 e 41 anos, com média de 25,2 anos de idade $(\mathrm{DP}=5,9)$, mediana e moda de 24 anos. No grupo estudado observou-se que, mais da metade das gestantes tinham menos de sete anos de estudo e conheceram seu status sorológico durante o prénatal; 70,0\% eram residentes em Fortaleza; mais de um terço $(35,5 \%)$ das infectadas tinham conhecimento sobre o diagnóstico de Aids antes de engravidar. Um elevado percentual das parturientes informou ter freqüentado serviço de pré-natal. (Tabela 1).

\section{Tabela 1}

Caracterização das parturientes infectadas pelo HIV segundo aspectos demográficos e epidemiológicos, em uma maternidade de referência. Fortaleza, Ceará, 2000 a 2004. $(n=110)$

\begin{tabular}{lcc}
\hline Variável & Número de casos & $\%$ \\
\hline Idade & & \\
$\quad 15-24$ anos & 58 & 52,7 \\
$25-41$ anos & & 47,3 \\
Escolaridade & 63 & 57,3 \\
0 a 7 anos de estudo & 47 & 42,7 \\
$>7$ anos de estudo & & \\
Município de residência & 77 & 70,0 \\
Fortaleza & 33 & 30,0 \\
Outros municípios & & \\
Conhecimento da sorologia & 45 & 40,9 \\
Antes do pré-natal & 63 & 57,3 \\
Durante o pré-natal & 2 & 1,8 \\
Durante o parto & & \\
Diagnóstico de Aids & 39 & 35,5 \\
Sim & 70 & 63,6 \\
Não & 1 & 0,9 \\
Ignorado & & 92,7 \\
Fez pré-natal & 102 & 5,5 \\
Sim & 6 & 1,8 \\
Não & 2 & \\
Ignorado & & \\
\hline & &
\end{tabular}


Tabela 2

Conhecimento da soropositividade para HIV pelas parturientes, segundo variáveis selecionadas em uma maternidade de referência. Fortaleza, Ceará, janeiro 2000 a agosto 2004.

\begin{tabular}{|c|c|c|c|c|c|}
\hline \multirow[t]{4}{*}{ Variável } & \multicolumn{4}{|c|}{ Conhecimento da Soropositividade } & \multirow{4}{*}{ OR (IC95\%) } \\
\hline & \multicolumn{4}{|c|}{ Sabiam ser soropositivo } & \\
\hline & \multicolumn{2}{|c|}{$\begin{array}{l}\text { Antes da gravidez } \\
\qquad(\mathrm{n}=45)\end{array}$} & \multicolumn{2}{|c|}{$\begin{array}{l}\text { Depois da gravidez } \\
\qquad(n=65)\end{array}$} & \\
\hline & $\mathrm{n}$ & $\%$ & $\mathrm{n}$ & $\%$ & \\
\hline \multicolumn{6}{|l|}{ Tipo de parto } \\
\hline Vaginal & 3 & 6,9 & 6 & 9,6 & 1,43 \\
\hline Cesáreo & 40 & 93,0 & 56 & 90,3 & $(0,29<O R<7,74)$ \\
\hline \multicolumn{6}{|c|}{ Profilaxia no pré-natal } \\
\hline Sim & 41 & 91,1 & 53 & 88,3 & 1,35 \\
\hline Não & 4 & 8,8 & 7 & 11,6 & $(0,32<O R<5,97)$ \\
\hline Profilaxia no parto & & & Ns & & \\
\hline $\operatorname{sim}$ & 42 & 97,6 & 57 & 89,0 & Ns \\
\hline Não & 1 & 2,3 & 7 & 10,9 & \\
\hline \multicolumn{6}{|c|}{ Profilaxia no pós-parto } \\
\hline Sim & 42 & 95,4 & 59 & 92,1 & 1,78 \\
\hline Não & 2 & 11,3 & 5 & 7,8 & $(0,29<O R<13,9)$ \\
\hline \multicolumn{6}{|l|}{ Aleitamento } \\
\hline Sim & 1 & 2,2 & 4 & 6,2 & 2,93 \\
\hline Não & 44 & 97,7 & 60 & 93,7 & $(0,29<O R<71,3)$ \\
\hline \multicolumn{6}{|l|}{ Adesão completa } \\
\hline $\operatorname{Sim}$ & 38 & 84,4 & 48 & 73,8 & 1,92 \\
\hline Não & 7 & 15,6 & 17 & 26,2 & $(0,66<O R<5,74)$ \\
\hline
\end{tabular}

Os resultados apontam uma proporção de 93,6\% de parturientes que fizeram uso de ARV no pré-natal e de $90,0 \%$ que utilizaram ARV no momento do parto. Nos recém-nascidos, a utilização de ARV nas primeiras 24 horas foi de $92,7 \%$, enquanto a suspensão do aleitamento materno foi de $96,2 \%$. A cesárea eletiva foi observada em $88,1 \%$ dos partos.

Não se encontrou diferença significativa entre o grupo de mulheres que tiveram conhecimento da soropositividade antes da gravidez com aquelas que souberam depois, em relação à utilização das seguintes medidas profiláticas: profilaxia com ARV no pré-natal, parto e recém-nascido, suspensão do aleitamento e parto cesáreo (Tabela 2).

Observou-se que a adesão às intervenções para a redução da transmissão vertical do HIV foi completa em $86(78,1 \%)$ dos casos. A freqüência de adesão foi maior entre aquelas que se sabiam soropositivas antes da gravidez $38(84,4 \%)$ do que entre as que souberam depois $48(73,8 \%)$. Os resultados mostraram não haver diferença estatisticamente significativa entre adesão e conhecimento da soropositividade antes ou depois da gravidez. (OR: 1,92; IC95\%: 0,66-5,74; $p=0,27$ ) (Tabela 2).

Entre as mulheres que tiveram adesão às intervenções completas, mais da metade $(56,9 \%)$ referiram ter até sete anos de estudo. No entanto, os resultados mostraram não haver associação entre escolaridade e adesão às medidas profiláticas. (OR: 1,06; IC95\%: 0,39-2,92; $p=0,90)$. Porém, o percentual de mulheres com grau de escolaridade com até sete anos de estudo foi maior $(55,5 \%)$ entre o grupo de parturientes que sabia ser soropositiva antes da gravidez. Não se encontrou diferença significativa entre o grupo de mulheres com conhecimento da soropositividade antes da gravidez com aquelas que souberam depois em relação à escolaridade, sendo o teste inconclusivo. (OR: 0,71; IC95\%: 0,30-1,65; $p=0,49)$. 


\section{Discussão}

Constatou-se que mais da metade das parturientes estudadas tinham em média 25 anos de idade e apresentavam baixa escolaridade, perfil semelhante ao encontrado nos resultados de Turchi et al. ${ }^{17} \mathrm{em}$ Goiânia, Goiás, onde a idade das parturientes variou entre 14 e 44 anos e média de 25 anos de idade. O perfil das parturientes infectadas pelo vírus HIV revela também ser o mesmo encontrado nos registros de Aids em mulheres do PN DST e Aids do Ministério da Saúde, desde o início da epidemia, apontando a vulnerabilidade e maior risco de adoecer na população atingida, que é de adulto jovem, com idade entre 20 e 39 anos e menor grau de escolaridade. 3

Com relação ao conhecimento das gestantes quanto ao seu status infeccioso, constatou-se que mais de $50 \%$ das mulheres se descobriram infectadas durante o pré-natal, período no qual a redução da transmissão vertical do HIV pode ser alcançada de forma mais efetiva, caso haja o diagnóstico precoce da infecção nesse período, o que ressalta a importância das ações na rede de atenção básica.7,18

Com relação à cobertura da sorologia para HIV, este estudo refere que mais de $90 \%$ das parturientes haviam sido testadas para infecção pelo HIV antes do parto. Resultados do estudo de Souza et al.,19 revelaram que no Brasil, a cobertura da sorologia para o HIV na gestação, levando em consideração todas as etapas antes do parto (pelo menos uma consulta de pré-natal; pedido de teste de HIV e conhecimento do resultado antes do parto), foi estimada em $52 \%$ e quando estratificada por regiões, o Nordeste ficou em torno de $24 \%$ e o Sul em $72 \%$, Estes percentuais divergem em diversas regiões do Brasil em relação à cobertura de testes anti-HIV no pré-natal, o que dificulta as intervenções para a redução da transmissão vertical do HIV.

Dessa forma, a realização de aconselhamento pré e pós-teste 20 torna-se de fundamental importância para a adesão às medidas de intervenção, tanto durante o pré-natal quanto em maternidades que abordam parturientes que perderam a oportunidade de realizar a sorologia para o HIV ou que não realizaram pré-natal.

Para a redução da transmissão vertical do HIV, os requisitos básicos para a implementação de ações em nosso meio incluem: acessibilidade e utilização de serviços de saúde para o período pré-natal, intraparto e pós-parto com profissionais de saúde devidamente treinados; serviços de aconselhamento pré e pós-teste; oferecimento de testes de HIV confiáveis e sem custo; laboratório equipado e apropriado para monitorar parâmetros sangüíneos relacionados à infecção pelo HIV. No Brasil, embora as intervenções visando à prevenção da transmissão vertical do HIV estejam disponíveis a todas as gestantes HIV positivas, as dificuldades do sistema único de saúde em prover diagnóstico laboratorial e as dificuldades para a obtenção do resultado, além do baixo percentual de mulheres testadas durante o pré-natal, principalmente nas populações mais vulneráveis, 6,21 e a qualidade do pré-natal aquém do desejáve16,18 comprometem a redução da taxa de transmissão vertical do Brasil. Um estudo com realizado por Vasconcelos e Hamann, 21 revelou que cerca de $24 \%$ e $27 \%$, das gestantes não tiveram acesso sequer ao AZT oral; $19 \%$ e $10 \%$ das parturientes não receberam o AZT intravenoso; $8 \%$ e 7\% dos bebês não foram medicados com o AZT solução oral, tendo os autores, apontado falhas importantes na implementação das ações, apesar da disponibilidade dos insumos. O coeficiente de transmissão vertical foi de $5,6 \%$, variando de $2,9 \%$ a $7,5 \%$, e foi maior no parto vaginal $(8 \%)$ e na cirurgia cesariana não-eletiva (7\%). Outro estudo de Nishimoto et al.,22 mostrou que a implantação de medidas preventivas tem importância na prevenção da transmissão do HIV mãe-filho, com redução de quase $40 \%$ no risco dessa transmissão comparada à encontrada anteriormente sem a utilização de medidas preventivas. Outros estudos documentaram estas dificuldades em outras partes do país, segundo Ramos et al.,23 inclusive em países de alta renda. ${ }^{13,14}$

Em Fortaleza, nas diferentes unidades de saúde, de referência ou não, foram identificadas falhas em várias etapas das estratégias de controle da transmissão vertical do HIV; encontrou-se elevado número de mulheres que não tiveram acesso às diferentes estratégias para redução da transmissão vertical, especialmente entre aquelas que engravidaram sem conhecimento de seu status sorológico,24,25 diferentemente do que foi constatado neste estudo. Provavelmente, isso se deva ao fato de tratar-se de uma unidade de referência em área central, com profissionais capacitados para a prevenção da transmissão vertical.

Esta investigação realizada em uma dessas unidades, evidencia que após quatro anos dessas iniciativas para a prevenção da transmissão vertical da infecção pelo HIV, a aplicação das intervenções vem sendo realizada de forma satisfatória, quando analisadas as 110 parturientes no estudo. Entretanto, como unidade de saúde de referência, pode ter justificado o fato de $57,3 \%$ das mulheres já saberem estar infectadas pelo HIV, 35,5\% delas com Aids manifesta. Chama a atenção o grande percentual de 
mulheres que referiu ter freqüentado serviço prénatal, reiterando a importância desse serviço.

O fato de essa maternidade ser uma unidade de referência pode justificar também a elevada adesão às ações de intervenção para a prevenção da transmissão vertical do HIV, o que não permite inferência para a situação geral do município de Fortaleza. Além disso, considerando-se o momento de conhecimento da soropositividade à infecção pelo HIV, não se encontrou diferença entre o grupo de mulheres que sabia o seu status sorológico antes da gravidez com as que não se sabiam infectadas em relação às medidas profiláticas e em relação à escolaridade. Também não se encontrou associação entre as mulheres com escolaridade maior ou menor que sete anos de estudo e adesão, diferentemente dos resultados do "estudo-sentinela parturiente" de Souza et al. 19 que evidenciaram disparidades sociais, representadas pelo gradiente decrescente de proporções por nível de escolaridade. Tais achados revelaram afunilamento acentuado na cobertura efetiva da detecção precoce da infecção pelo HIV na gestação, tanto maior quanto pior for o nível socioeconômico.

A transmissão vertical do HIV é um desafio para a saúde pública, demandando novas estratégias de vigilância, incorporando, de forma integrada, dados relativos ao processo infeccioso e ao comportamento da população.26,27 No Brasil, do ponto de vista da vigilância epidemiológica, é realizada a Notificação Compulsória de Gestantes Infectadas pelo HIV/ Crianças Expostas, desde 2000, e que permanece

\section{Referências}

1. Brasil. Ministério da Saúde. Programa Nacional de DST/Aids. Projeto Nascer-Maternidades. Brasília, DF; 2003.

2. WHO (World Health Organization). Joint United Nations Programme on HIV/AIDS 2003: Aids epidemic update. Geneva; 2004.

3. Brasil. Ministério da Saúde. Secretaria de Vigilância em Saúde. Programa Nacional de DST/Aids. Dados epidemiológicos-AIDS. Bol Epidemiol. 2004. 18: (1).

4. Ceará. Secretaria de Saúde. Infecções no trato reprodutivo no Estado do Ceará: documento base, texto preliminar. Fortaleza; 2000

5. Brasil. Ministério da Saúde. Secretaria de Políticas de Saúde. Coordenação Nacional de DST/Aids. Dados epidemiológicos do Brasil. Bol Epidemiol. 2002; 16: (01).

6. Veloso VG, Vasconcelos AL, Grinsztejn B. Prevenção da transmissão vertical do HIV no Brasil. Bol Epidemiol. 1999; 12: 16-23. como a principal estratégia, apesar da dificuldade de análise dos dados obtidos. ${ }^{2}$

Mesmo com todos os entraves, de uma forma geral, a incidência de casos de Aids em crianças por exposição vertical vem decrescendo progressivamente em nosso país nos últimos anos, apesar das diferenças regionais observadas. 26

Para uma análise mais ampliada da situação de Fortaleza, podem fazer-se necessários estudos com ênfase na atenção básica e nas unidades de atenção secundária - maternidades que não são referência para a infecção pelo HIV, e rede de atenção integral à gestante/parturiente - que os dados apresentados são um indicativo que muito pode ser alcançado. Grande parte das mulheres ainda não tem acesso ao teste de HIV, seja pela sua condição social, seja por falhas no sistema de saúde. A ausência de detecção precoce da infecção pelo HIV durante o pré-natal representa uma oportunidade perdida de intervenção na gestante infectada, limitando a possibilidade de redução da transmissão vertical do HIV. É a partir da estruturação dessa rede que inclui laboratórios, e do processo de referência e contra-referência, que os resultados encontrados nesse estudo poderão ser reproduzidos.

\section{Agradecimentos}

Ao Centro de Estudo de AIDS/DST do Rio Grande do Sul (CEARGS).
7. Brasil. Ministério da Saúde. Secretaria de Vigilância em Saúde. Programa Nacional de DST/Aids: recomendações para profilaxia da transmissão vertical do HIV e terapia anti-retroviral em gestantes: 2004. Brasília, DF; 2004.

8. Connor EM, Sperling RS, Gelber R, Kiselev P, Scott G, O'Sullivan MJ, VanDyke R, Bey M, Shearer W, Jacobson RL, Jimenez E, O'Neill E, Bazin B, Delfraissy J-F, Culnane M, Coombs R, Elkins M, Moye J, Stratton P, Balsley J; for the Pediatric AIDS Clinical Trials Group Protocol 076 Study Group. Reduction of maternal-infant transmission of human immunodeficiency virus type 1 with zidovudine treatment. pediatric AIDS Clinical Trials Group Protocol 076 Study Group. N Engl J Med. 1994; 331: 1173-80.

9. Lambert JS, Nogueira SA. Manual para o acompanhamento clínico da gestante infectada pelo HIV. Rio de Janeiro: Ministério da Saúde; 2002. 
10. Shaffer N, Chuachoowong R, Mock PA, Bhadrakom C, Siriwasin W, Young NL, Chotpitayasunondh T, Chearskul S, Roongpisuthipong A, Chinayon P, Karon J, Mastro TD, Simonds RJ; on behalf of the Bangkok Collaborative Perinatal HIV Transmission Study Group. Short-course zidovudine for perinatal HIV-1 transmission in Bangkok, Thailand: a randomised controlled trial. Lancet. 1999; 353: 773-80.

11. Lallemant M, Jourdain G, Le Coeur S, Kim S, Koetsawang S, Comeau AM, Phoolcharoen W, Essex M, McIntosh K, Vithayasai V. A trial of shortened zidovudine regimens to prevent mother-to-child transmission of human immunodeficiency virus type 1. Perinatal HIV Prevention Trial (Thailand ) Investigators. N Engl J Med. 2000; 343: 98291.

12. Nogueira SA, Abreu T, Oliveira R, Araújo L, Costa T, Andrade M, Psic MFG, Machado, Rodrigues K, Mercadante ER, Fernandes I, Sapia MC, Lambert JS. Successful prevention of HIV transmission from mother to infant in Brazil using a multidisciplinary team approach. Braz J Infect Dis. 2001; 5: 78-86.

13. CDC (Centers for Diseases Control and Prevention). Public health service recommendations for human immunodeficiency virus counseling and voluntary testing for pregnant women. Atlanta, GA. MMWR. 1995; 44 (RR-7): 1-15.

14. CDC (Centers for Diseases Control and Prevention). Recommendations of the public health service task force on use of zidovudine to reduce perinatal transmission of human immunodeficiency virus. Atlanta, GA. MMWR. 1994; 43 (RR-11):1-20.

15. Brasil. Ministério da Saúde. Programa Nacional de DST/Aids: recomendações para profilaxia da transmissão vertical do HIV e terapia anti-retroviral em gestantes. Brasília, DF; 2004.

16. Brasil. Ministério da Saúde. Secretaria de Políticas de Saúde. Área Técnica da Saúde da Mulher. Parto, aborto e puerpério: assistência humanizada à mulher. Brasília, DF; 2001.

17. Turchi MD, Duarte LS, Martelli CMT. Mother-to-child transmission of HIV: risk factors and missed opportunities for prevention among pregnant women attending health services in Goiânia, Goiás State, Brazil. Cad Saúde Pública. 2007; 23 (Supl 3): 390-401.

Recebido em 30 de abril de 2007

Versão final apresentada em 7 de setembro de 2008

Aprovado em 9 de novembro de 2008
18. Brasil. Ministério da Saúde. Secretaria de Políticas de Saúde. Área Técnica da Saúde da Mulher. Assistência prénatal: manual técnico. 3. ed. Brasília, DF; 2000.

19. Souza Jr PRB, Szwarcwald CL, Barbosa Jr A, Carvalho MF, Castilho EA. Infecção pelo HIV durante a gestação: estudo-sentinela parturiente, Brasil: 2002. Rev Saúde Pública. 2004; 38: 764-72.

20. Brasil. Ministério da Saúde. Secretaria de Políticas Públicas. Coordenação Nacional de DST/Aids. Aconselhamento em DST/HIV e Aids: diretrizes e procedimentos básicos. Brasília, DF; 1999.

21. Vasconcelos ALR, Hamann EM. Por que o Brasil ainda registra elevados coeficientes de transmissão vertical do HIV? Uma avaliação da qualidade da assistência prestada a gestantes/parturientes infectadas pelo HIV e seus recémnascidos. Rev Bras Saúde Matern Infant. 2005; 5: 483-92.

22. Nishimoto TMI, Eluf Neto J, Rozman MA. Transmissão materno-infantil do vírus da imunodeficiência humana: avaliação de medidas de controle no município de santos. Rev Assoc Med Bras. 2005; 51: 54-60

23. Ramos MC, Gregol, LRG, Germany C, Silva JVB, Sander MA, Rutherford G. Prevention of mother-to-children transmission of HIV: compliance with the recomendations of de Brazilian National STD/Aids control program for prenatal and and perinatal HIV testing in Porto Alegre, Brazil. AIDS Behav. 2002; 6: 277-82.

24. Cavalcante MS. Gestantes infectadas pelo HIV: análise das condutas utilizadas na prevenção da transmissão vertical do vírus da imunodeficiência humana (HIV) em Fortaleza, Ceará, no período de 1999 a 2001 [dissertação]. Fortaleza: Departamento de Saúde Comunitária, Faculdade de Medicina da Universidade Federal do Ceará; 2003.

25. Cavalcante MS, Ramos Jr NA, Silva TMJ, Kerr-Pontes LRS. Transmissão vertical do HIV em Fortaleza: revelando a situação epidemiológica em uma capital do Nordeste. Rev Bras Ginecol Obstet. 2004; 26: 131-8.

26. Brasil. Ministério da Saúde. Secretaria de Políticas de Saúde. Coordenação Nacional de DST e Aids. Vigilância do HIV no Brasil: novas diretrizes. Brasília, DF; 2002.

27. UNAIDS (Joint United Nations Programme on HIV/Aids). WHO (World Health Organization): guidelines for second generation HIV surveillance: the next decade. Geneva; 2000. 